\title{
Transplant restoration of spinal cord inhibitory controls ameliorates neuropathic itch
}

\author{
Joao M. Braz, ${ }^{1}$ Dina Juarez-Salinas, ${ }^{1}$ Sarah E. Ross, ${ }^{2}$ and Allan I. Basbaum ${ }^{1}$ \\ 'Department of Anatomy, UCSF, San Francisco, California, USA. ²Department of Neurobiology, University of Pittsburgh, Pittsburgh, Pennsylvania, USA.
}

\begin{abstract}
The transmission of pruritoceptive (itch) messages involves specific neural circuits within the spinal cord that are distinct from those that transmit pain messages. These itch-specific circuits are tonically regulated by inhibitory interneurons in the dorsal horn. Consistent with these findings, it has previously been reported that loss of GABAergic interneurons in mice harboring a deletion of the transcription factor Bhlhb5 generates a severe, nonremitting condition of chronic itch. Here, we tested the hypothesis that the neuropathic itch in BHLHB5-deficient animals can be treated by restoring inhibitory controls through spinal cord transplantation and integration of precursors of cortical inhibitory interneurons derived from the embryonic medial ganglionic eminence. We specifically targeted the transplants to segments of the spinal cord innervated by areas of the body that were most severely affected. BHLHB5-deficient mice that received transplants demonstrated a substantial reduction of excessive scratching and dramatic resolution of skin lesions. In contrast, the scratching persisted and skin lesions worsened over time in sham-treated mice. Together, these results indicate that cell-mediated restoration of inhibitory controls has potential as a powerful, cell-based therapy for neuropathic itch that not only ameliorates symptoms of chronic itch, but also may modify disease.
\end{abstract}

\section{Introduction}

Recent studies indicate that independent dorsal horn circuits transmit the pruritoceptive and nociceptive information that generates itch and pain (1-9). For example, although primary afferent nociceptors that express the capsaicin receptor TRPV1 convey both noxious and pruritic stimuli to the spinal cord dorsal horn, recent evidence indicates that different spinal circuits are engaged. In particular, itch occurs when a population of gastrin-releasing peptide receptor-expressing interneurons in the superficial dorsal horn are activated. Indeed, deletion of these interneurons eliminates scratching provoked by a variety of pruritogens, without altering behaviors provoked by painful stimuli. Not only do itch and pain appear to arise from activation of distinct afferent and spinal cord circuits, but they are also mutually interfering. Thus, painful stimulation (e.g., scratching) reduces itch, and while morphine reduces pain, it can trigger itch (10). However, both pruritoceptive and nociceptive circuits are regulated by GABAergic inhibitory interneurons in the dorsal horn, and loss of these controls contributes to chronic neuropathic itch and pain (11-15). Chronic itch is particularly noticeable in mice with a deletion of the gene that encodes BHLHB5, an atonal-related transcription factor. In these mice, there is a dramatic loss of dorsal horn GABAergic interneurons, which underlies a neuropathic itch phenotype manifest as self-inflicted skin lesions that result from excessive licking and scratching (12).

Recently, we reported that transplants of GABAergic progenitor cells derived from the embryonic medial ganglionic eminence (MGE) of the mouse cerebral cortex into the spinal cord of adult

Conflict of interest: The authors have declared that no conflict of interest exists. Submitted: January 14, 2014; Accepted: May 29, 2014.

Reference information: J Clin Invest. 2014;124(8):3612-3616. doi:10.1172/JCI75214. mice functionally integrate into host circuits and relieve the mechanical hypersensitivity produced in a neuropathic pain model (16). As loss of GABAergic interneurons contributes to the chronic itch that characterizes the Bhlhb5 mutant mice, here we assessed the utility of "replenishing" the pool of spinal cord inhibitory interneurons to treat neuropathic itch.

\section{Results and Discussion}

Bhlhb5 mutant mice are in poor general health. They eat and drink less than their WT littermates, and most mice die before or are euthanized between 8 and 10 weeks of age. Skin lesions appear around 4 weeks, most commonly in the perineum, but also in the haunches and occasionally in orofacial regions. The skin lesions progressively worsen and never resolve spontaneously. Here, we attempted to ameliorate this chronic itch condition by restoring inhibitory control to the spinal segments that receive afferent input from areas with skin lesions. To this end, in mice with lesions of the haunches, we transplanted MGE cells into lumbar and rostral sacral segments. In mice with neck or orofacial skin lesions, we transplanted cells into the upper cervical spinal cord and trigeminal nucleus caudalis, respectively. Most commonly, we transplanted MGE cells at around 5 to 6 weeks of age, at which point moderate to severe skin lesions were documented. Because most lesions are unilateral, MGE cells were transplanted in the dorsal horn ipsilateral to the lesions. We did not observe any effect on the contralateral side, which was devoid of lesions. The severity of the lesions was monitored over time. Control mice received injections of medium, without cells.

Within 2 weeks of the transplant, we observed a significant reduction in the size and severity of the skin lesions in the hind limb (Figure 1, A-C) or neck (Figure 1, D-F), ipsilateral to the trans- 

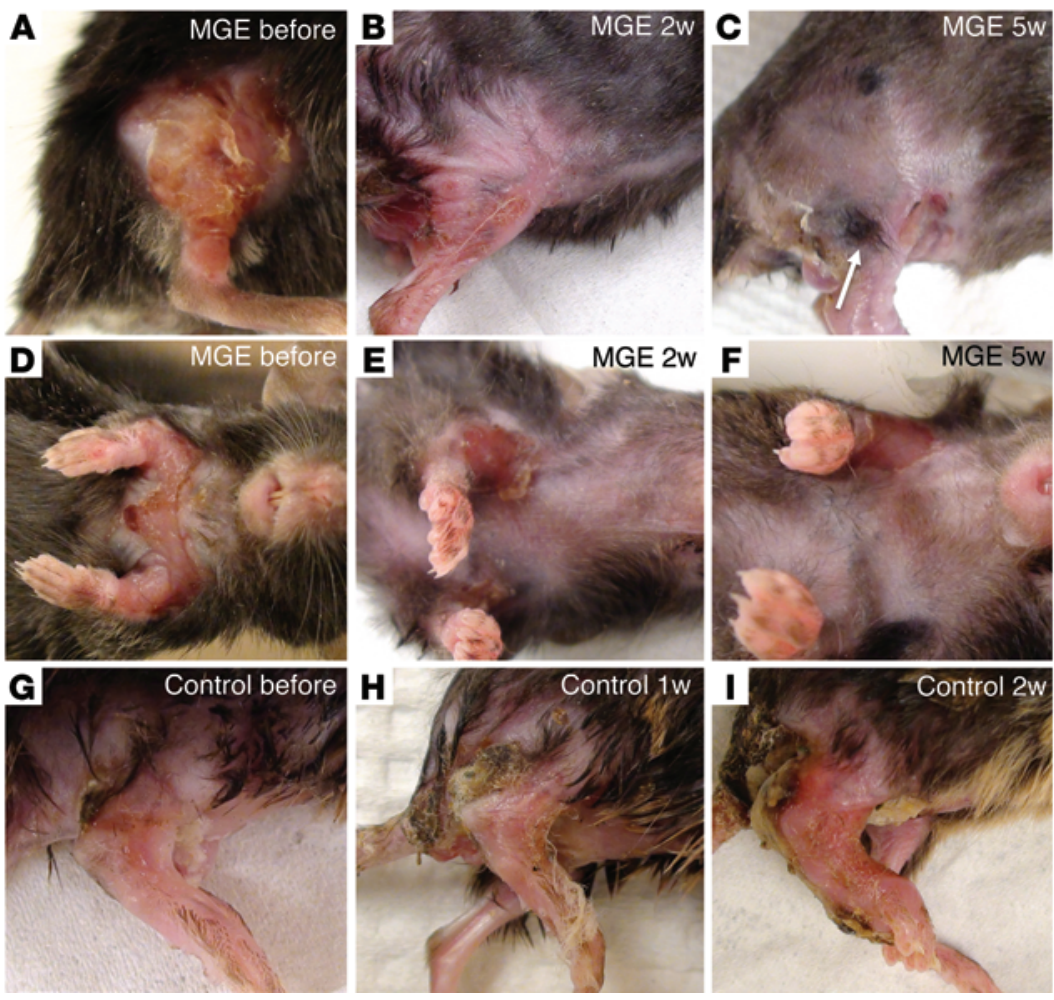

planted spinal segment. The extent and severity of the lesions decreased over time, completely disappearing in some animals within 5 weeks (Figure 1, C and F). In some cases, we observed new hair growth in the previously denuded region (Figure 1C). In contrast, we never observed recovery in control mice (Figure 1, G-I). In fact, the progressive deterioration in control mice typically required their euthanization, often within 2 to 3 weeks of the medium transplant.

To determine whether MGE transplant-induced skin-lesion improvement correlated with reduced itch, in a separate group of mice, we monitored spontaneous scratching, licking, and biting. At 2 and 3 weeks after transplantation, we recorded the number of scratches over a 30-minute period. Figure 2 shows that MGE transplants significantly reduced these behaviors, by approximately $70 \%$ at 2 weeks after transplantation. In contrast, scratching, licking, and biting in control mice continued unabated. Three weeks after transplantation, these behaviors remained low in MGE-transplanted mice (data not shown), but unexpectedly, were also reduced in control animals. However, as control mice died or euthanization was mandated either before or soon after this time point, we believe that the reduced behaviors related to the poor health of the control mice.

Figure 3, A-C, illustrates that MGE cells thrived in the spinal cord of the Bhlhb5 mutant mice. We determined that approximately $5 \%(5.1 \% \pm 3.1 \% ; n=5)$ of MGE cells survived 4 weeks after transplantation. NeuN double labeling confirmed that the majority $(\sim 80 \%)$ of transplanted cells differentiated into neurons (Figure 3C) and expressed GABA (Figure 3, D-F). Some MGE cells expressed parvalbumin or neuropeptide $\mathrm{Y}$, markers of subpopulations of inhibitory interneurons (data not shown). Double labeling for GFP and somatostatin (SST) (Figure 3, G-I),
Figure 1. Intraspinal transplantation of GABAergic precursor (MGE) cells resolves skin lesions in Bhlhb5 mutant mice. (A-F) Two representative examples of $B h / h b 5$ mutant mice before ( $\mathbf{A}$ and $\mathbf{D}), 2$ weeks after (B and $\mathbf{E})$, and 5 weeks after (C and $\mathbf{F}$ ) MGE transplantation into the lumbar $(\mathbf{A}-\mathbf{C})$ and cervical spinal cord (D-F). There is remarkable, progressive improvement of the affected region ipsilateral to the transplant. In some cases, we observed new hair growth in previously denuded regions (arrow in C). In contrast, skin lesions in animals that received only medium (G-I) progressively worsened. All mice were 5 to 6 weeks of age at the time of transplant. Because of the deterioration of control animals, euthanization was generally required by 7 to 8 weeks of age.

a cotransmitter in cortical but not spinal cord GABAergic neurons, revealed that some MGE cells also expressed SST, which suggests that the transplanted cells also retained their cortical phenotype even in the context of chronic itch.

To assess functional integration of the MGE transplants 3 weeks after transplantation, we injected dilute formalin, a noxious chemical stimulus, into the hind paw of the Bhlhb5 mutant mice. We used Fos expression as a general neuronal activity marker. Figure 3, J-L, shows that formalin induced expression of Fos in both host and $\mathrm{GFP}^{+}$ transplanted MGE cells. This result indicates that the cells have integrated into the host circuitry and received primary afferent inputs. Finally, as our previous studies demonstrated that MGE transplants can normalize dorsal horn levels of the biosynthetic enzyme for GABA, namely glutamic acid decarboxylase (GAD65 and GAD67), we also examined GAD levels before and after transplant in the Bhlhb5 mutant mice. Consistent with previous reports showing that a large number of inhibitory interneurons are lost in the spinal cord of the Bhlhb5 mutant mice (17), we recorded a dramatic reduction in GAD enzyme levels (GAD67-WT: $0.36 \pm 0.03 \mathrm{AU}$; GAD67-Bhlhb5: $0.16 \pm 0.01 \mathrm{AU}(-54 \%)$; GAD65WT: $0.18 \pm 0.06 \mathrm{AU}$; GAD65-Bhlhb5: $0.06 \pm 0.03 \mathrm{AU}(-65 \%)$; WT: $n=3$; Bhlhb5: $n=4)$. Surprisingly, these levels did not differ in MGE-transplanted Bhlhb5 mutant mice (GAD67: $0.15 \pm 0.01 \mathrm{AU}$; GAD65: $0.07 \pm 0.03 \mathrm{AU} ; n=4)$. The failure of transplants to normalize GAD levels in the Bhlhb5 mutant mice contrasts sharply with what we observed in transplanted mice that underwent nerve injury (16). An important difference in the 2 conditions is that the decrease of GAD levels following nerve injury was rather small $(-10 \%)$. We presume that the number of transplanted MGE transplants was not sufficient to overcome the very large decrease in GAD levels in the Bhlhb5 mutant mice. The fact that only a small number of transplanted cells is sufficient to restore inhibitory control is presumably related to the remarkable axonal arborization of the transplanted neurons (Figure 3, A and B).

Traditional approaches to treating chronic pruritus that target peripheral mediators are far from satisfactory. For this reason, recent studies focused on identifying the central neural circuits through which itch is generated. For example, there is evidence that spinal cord disinhibition of itch signaling due to a loss of inhibitory interneurons (12) or decreased primary afferent 


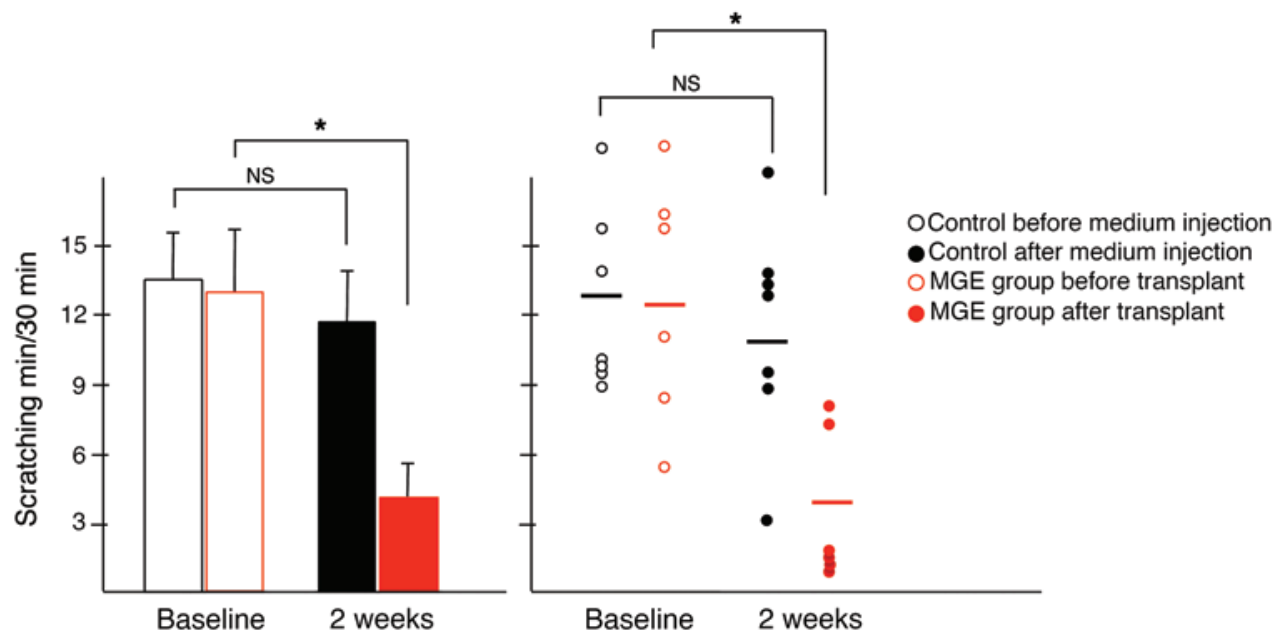

Figure 2. Intraspinal transplantation of GABAergic precursor (MGE) cells reduces excessive scratching, biting, and licking in Bhlhb5 mutant mice. Bhlhb5 mutant mice exhibit excessive licking, biting, and scratching (baseline). This spontaneous behavior usually developed around 4 weeks of age. Two weeks after transplantation, these behaviors were significantly reduced in mice that received MGE cells (red, $n=6 ;-70 \%$, $\left.{ }^{*} P<0.05\right)$. In contrast, these behaviors persisted in control animals (black, $n=7 ;-12 \%$ NS). Scratching behavior was measured in minutes over a 30-minute period of observation. The illustration to the right displays the individual data points.

drive (18) can enhance spontaneous itch. Given that the Bhlhb5 mutant mice develop a chronic scratching condition in association with a loss of a large population of GABAergic inhibitory interneurons, it is likely that their condition models neuropathic itch, namely itch associated with direct damage to the central nervous system. In this respect, the itch is comparable to the neuropathic pain that can be triggered by dysfunctional dorsal horn GABAergic circuits. By replenishing a pool of inhibitory interneurons, MGE transplantation can overcome the loss of inhibition, thus mitigating the presumptive underlying etiology of the neuropathic itch condition. Not only did we demonstrate that there was significantly reduced scratching in MGE cell-transplanted mice, but we also observed remarkable healing of the previously affected region. Importantly, the area of skin that recovered corresponded to the spinal cord target of the transplant. In other words, the treatment does not result from a systemic response to mediators released from the transplant, but likely reflects local neuronal circuit reorganization. Indeed, when excessive scratching and lesions were present over multiple body regions, only the region that corresponded to the transplant was influenced. Interestingly, even though the loss of GABAergic neurons in the Bhlhb5 mutant mice occurs throughout the spinal cord, bilaterally, most lesions only develop on one side of the body, indicating that loss of inhibition is not sufficient to trigger exacerbated scratching.

Taken together with our previous results demonstrating utility of the transplants in a model of neuropathic pain, our present findings illustrate that there are commonalities in the pathophysiology of neuropathic itch and pain and in the approaches that can be taken in their management. Specifically, our results reinforce the idea that transplantation of GABAergic precursors has the essential properties for a cell-based therapy for nerve damage-associated chronic itch and pain, particularly when loss of inhibitory control is a major contributor to the condition. Whether this loss of inhibitory controls is a common pathophysiological mechanism in different models of chronic itch remains to be determined. But the fact that both GABA and glycine receptors antagonists block scratching-induced inhibition of spontaneous firing of dorsal horn neurons in a dry skin model of chronic itch (11) suggests that transplants may also be effective in chronic itch conditions that do not arise from nerve damage. Consistent with this possibility, withdrawal of intrathecal baclofen, a GABA-B agonist, provokes severe pruritus in patients (19), presumably because of an acute loss of inhibitory control.

However, as the present findings involve transplants of embryonic cortical tissue, it is not clear that MGE transplants can be translated directly to the clinic. Transplants of human fetal MGE cells can be envisioned, but this approach unquestionably comes with considerable logistic and ethical limitations. Recent advances with embryonic or induced pluripotent stem cells that differentiate into functional MGE-like GABAergic interneuron subtypes, however, provide a promising and powerful alternative approach $(20,21)$. Importantly, as we demonstrated for the MGE-derived GABAergic cells, there is robust synaptic integration of the human cells into host circuitry. Furthermore, we emphasize that transplantation of GABAergic precursors is not equivalent and indeed is likely superior to spinally administered GABAergic pharmacotherapy. Transplanted cells are not equivalent to an intrathecal GABAergic pump, which nonspecifically blocks all spinal cord circuits. Rather, transplanted cells integrate into local circuits and thus serve to repair disrupted GABAergic circuits. For this reason, the transplant-mediated local delivery of inhibitory neurotransmitters should also avoid many of the adverse side effects associated with current systemically administered pharmacotherapies for the treatment of chronic itch.

\section{Methods}

Animals and transplantation procedure. MGE cells were transplanted into Bhlhb5 mutant male mice ( 5 to 6 weeks old), as previously described (16). One group received MGE cells; the control group received medi- 

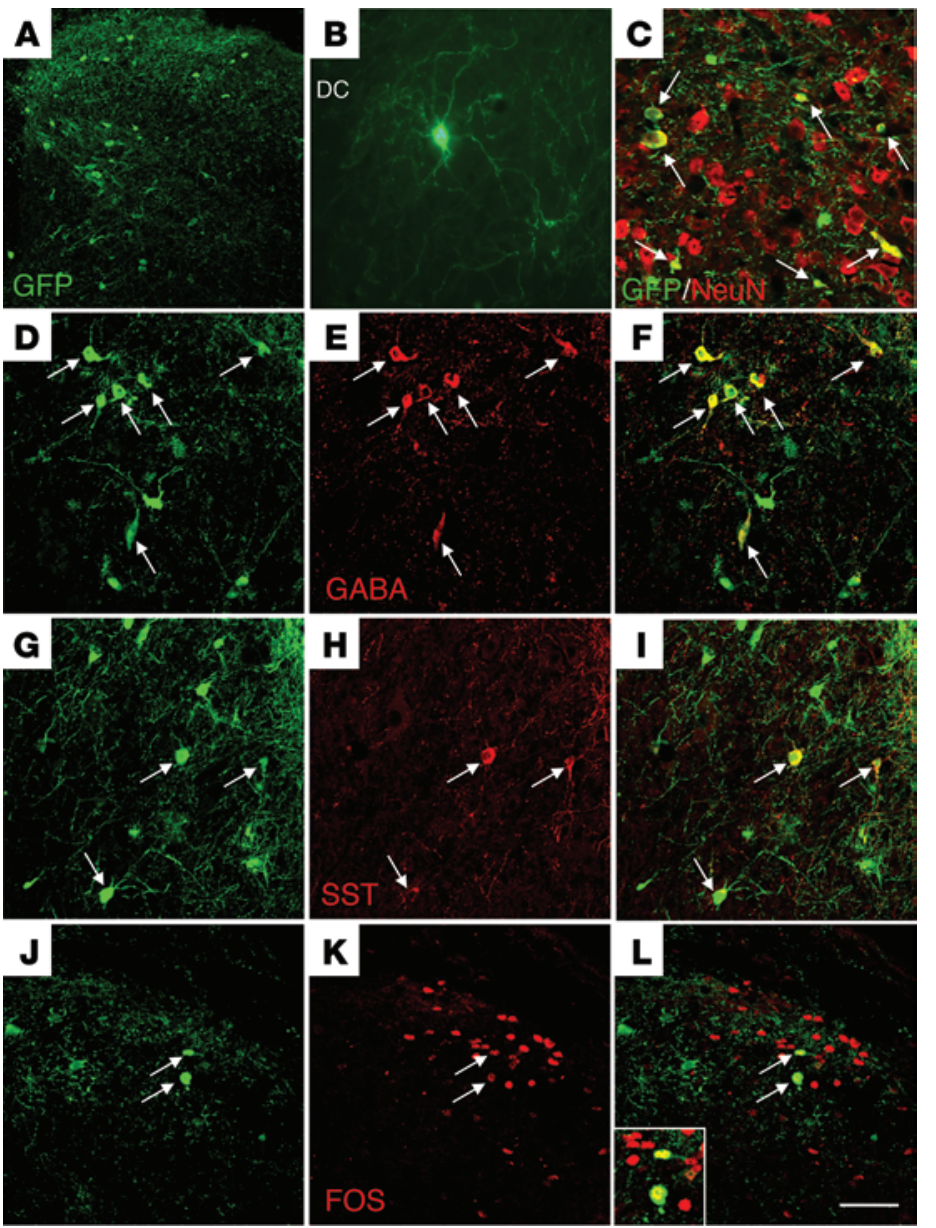

um. Cells (approximately 50,000) were transplanted over 2 spinal cord segments, depending on the skin-lesion location. To "treat" lesions of the hind limbs, we transplanted MGE cells into ipsilateral lumbar and rostral sacral spinal cord. For neck lesions, cells were injected into cervical spinal cord (between C4 and C8). For orofacial lesions, cells were transplanted in trigeminal nucleus caudalis. In animals with bilateral lesions of the thorax, we injected cells on both sides of the cord. Survival rates were similar for all injection sites. Sixty-five percent of animals did not survive the first week after surgery, regardless of treatment, presumably because of the poor health of the mice. Importantly, in all MGE-transplanted mice that survived the first week $(n=13)$, we were able to document progressive improvement of the itch phenotype. In contrast, none of the surviving control mice $(n=7)$ showed skin-lesion improvement. In a subgroup of these animals, we documented spontaneous scratching before and once a week after transplantation.

Antibodies and immunohistochemistry. Antibodies were as follows: rabbit anti-GFP (1:2000; Molecular Probe), mouse anti-NeuN (1:2000; Chemicon), chicken anti-GFP (1:2000; Abcam), rabbit anti-GABA
Figure 3. Transplanted MGE progenitor cells differentiate into GABAergic interneurons and integrate into host circuitry of the Bhlhb5 mutant mice. (A-C) GFP-expressing (green) MGE cells in the spinal cord dorsal horn of Bhlhb5 mutant mice. Cells dispersed throughout the dorsal horn (A), extended long processes (B), and the great majority expressed the neuronal marker NeuN (C). DC, dorsal column. (D-F) Most of the GFP+ cells also express GABA. (G-I) Some GFP+ cells coexpress SST, indicating that the MGE transplants retain characteristics of cortical GABAergic interneurons. (J-L) A peripheral noxious stimulus (hind paw injection of dilute formalin) induces expression of Fos, a marker of neuronal activity, in GFP+ (i.e., transplanted) cells. Arrows point to double-labeled cells. Inset in L illustrates $2 \mathrm{GFP}^{+} \mathrm{Fos}^{+}$double-labeled cells. Scale bar: $150 \mu \mathrm{m}$ (A); $50 \mu \mathrm{m}$ (B-L); $25 \mu \mathrm{m}$ (inset).

(1:2000; Sigma-Aldrich), and rabbit anti-SST (1:500, Peninsula). Immunohistochemistry was performed as previously described (16).

Behavioral analysis. Animals were placed individually in Plexiglass cylinders, and spontaneous scratching, licking, and biting were monitored by video recording over 30 minutes, once before and once a week after transplantation, for up to 3 weeks. The investigator scoring the behavior was blind to treatment. Only surviving and successfully transplanted animals (with at least $1 \mathrm{GFP}^{+}$cell per section through the transplanted spinal cord segment) were included in the statistical analyses.

Quantitative PCR. Bhlhb5 mutant mice were transplanted with medium $(n=4)$ or MGE cells $(n=4)$. Naive, nontransplanted WT mice $(n=3)$ were used as controls. Three weeks after transplantation, the transplanted segment of spinal cord and a comparable region from control animals were dissected, after which the cord was divided into ipsilateral and contralateral halves (containing both dorsal and ventral horn tissue). We used TRIzol to extract mRNA. Next, 200 ng of purified mRNA was reverse transcribed into cDNA using Superscript II (Invitrogen). Gad65, Gad67, and $\beta$-actin mRNA were quantified with a CFX connect real-time PCR system (BioRad) using SYBR Green PCR Master Mix (Applied Biosystems). Ratios of Gad65 and Gad67 to $\beta$-actin mRNA were compared and analyzed by 2-way ANOVA.

Statistics. Data are expressed as mean \pm SEM; $n$ indicates the number of mice. Raw data were analyzed by Student's $t$ test (1-tailed).

Study approval. All experiments were approved by the UCSF IACUC.

\section{Acknowledgments}

This work was supported by grants from the NIH (NS78326, NS14627, AR059402).

Address correspondence to: Joao M. Braz, University of California San Francisco, Rock Hall Building, 1550 4th Street, Room 345, San Francisco, California 94158, USA. Phone: 415.476.4311; E-mail: bjoao@phy.ucsf.edu.
1. Akiyama T, Carstens E. Neural processing of itch. Neuroscience. 2013;250:697-714.

2. Liu Q, et al. Sensory neuron-specific GPCR Mrgprs are itch receptors mediating chloroquineinduced pruritus. Cell. 2009;139(7):1353-1365.

3. Kim SJ, et al. Analysis of cellular and behavioral responses to imiquimod reveals a unique itch pathway in transient receptor potential vanilloid 1 (TRPV1)-expressing neurons. Proc Natl Acad Sci U S A. 2011;108(8):3371-3376.

4. Han L, et al. A subpopulation of nociceptors specifically linked to itch. Nat Neurosci. 2013;16(2):174-182.

5. Sun YG, Chen ZF. A gastrin-releasing peptide receptor mediates the itch sensation in the spinal cord. Nature. 2007;448(7154):700-703.

6. Mishra SK, Hoon MA. The cells and circuitry for itch responses in mice. Science. 2013;340(6135):968-971.

7. Imamachi N, et al. TRPV1-expressing primary afferents generate behavioral responses to pruri- 
togens via multiple mechanisms. Proc Natl Acad SciU S A. 2009;106(27):11330-11335.

8. Wang X, et al. Excitatory superficial dorsal horn interneurons are functionally heterogeneous and required for the full behavioral expression of pain and itch. Neuron. 2013;78(2):312-324.

9. Braz JM, Solorzano C, Wang X, Basbaum AI. Transmitting pain and itch message: a contemporary of the spinal cord circuits that generate Gate Control. Neuron. 2014;82(3):522-536.

10. Liu XY, et al. Unidirectional cross-activation of GRPR by MOR1D uncouples itch and analgesia induced by opioids. Cell. 2011;147(2):447-458.

11. Akiyama T, Iodi Carstens M, Carstens E. Transmitters and pathways mediating inhibition of spinal itch-signaling neurons by scratching and other counterstimuli. PLoS One. 2011;6(7):e22665.

12. Ross SE, et al. Loss of inhibitory interneurons in the dorsal spinal cord and elevated itch in Bhlhb5 mutant mice. Neuron. 2010;65(6):886-898.

13. Moore KA, Kohno T, Karchewski LA, Scholz J, Baba H, Woolf CJ. Partial peripheral nerve injury promotes a selective loss of GABAergic inhibition in the superficial dorsal horn of the spinal cord. J Neurosci. 2002;22(15):6724-6731.

14. Lever I, Cunningham J, Grist J, Yip PK, Malcangio M. Release of BDNF and GABA in the dorsal horn of neuropathic rats. Eur J Neurosci. 2003;18(5):1169-1174.

15. Ibuki T, Hama AT, Wang XT, Pappas GD, Sagen J. Loss of GABA-immunoreactivity in the spinal dorsal horn of rats with peripheral nerve injury and promotion of recovery by adrenal medullary grafts. Neuroscience. 1997;76(3):845-858.

16. Braz JM, et al. Forebrain GABAergic neuron precursors integrate into adult spinal cord and reduce injury-induced neuropathic pain. Neuron. 2012;74(4):663-675.
17. Kardon AP, et al. Dynorphin acts as a neuromodulator to inhibit itch in the dorsal horn of the spinal cord. Neuron. 2014;82(3):573-586.

18. Liu Y, et al. VGLUT2-dependent glutamate release from nociceptors is required to sense pain and suppress itch. Neuron. 2010;68(3):543-556.

19. Ben Smail D, Hugeron C, Denys P, Bussel B. Pruritus after intrathecal baclofen withdrawal: a retrospective study. Arch Phys Med Rehab. 2005;86(3):494-497.

20. Nicholas CR, et al. Functional maturation of hPSC-derived forebrain interneurons requires an extended timeline and mimics human neural development. Cell Stem Cell. 2013;12(5):573-586.

21. Chambers SM, Fasano CA, Papapetrou EP, Tomishima M, Sadelain M, Studer L. Highly efficient neural conversion of human ES and iPS cells by dual inhibition of SMAD signaling. Nat Biotechnol. 2009;27(3):275-280. 